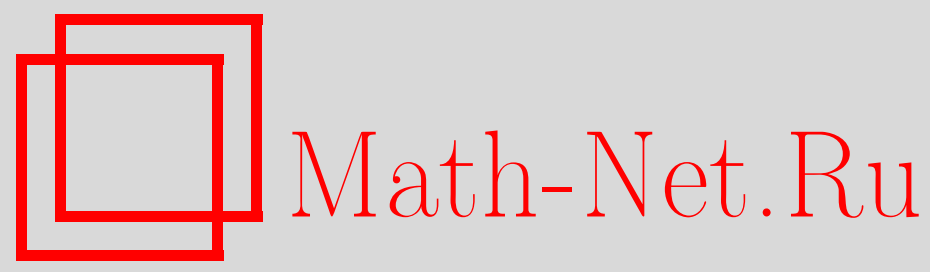

C. А. Степанянц, Лакунарные условия тауберова типа для методов суммирования Чезаро, Матем. заметки, 1999, том 65, выпуск 1, 118-129

DOI: https://doi.org/10.4213/mzm1033

Использование Общероссийского математического портала Math-Net.Ru подразумевает, что вы прочитали и согласны с пользовательским соглашением http://www . mathnet.ru/rus/agreement

Параметры загрузки:

IP: 18.209 .158 .208

26 апреля 2023 г., $14: 37: 58$

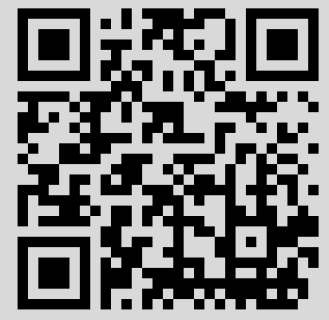




\section{ЛАКУНАРНЫЕ УСЛОВИЯ ТАУБЕРОВА ТИПА ДЛЯ МЕТОДОВ СУММИРОВАНИЯ ЧЕЗАРО}

\section{С. А. Степанянц}

В статье изучаются лакунарные условия, обеспечивающие на классе рядов, им удовлетворяющим, эквивалентность методов суммирования Чезаро $(C, k)$ и $(C, \alpha)$ с фиксированными $k$ и $\alpha(0<k<\alpha)$. Установлена достаточность условия, в котором "лакуна" соответствующей величины разделяет не соседние ненулевые элементы (как в классическом случае $k=0$ ), но ограниченные в совокупности множества из $k+1$ ненулевых элементов.

Библиограффия: 6 названий.

1. В данной работе рассматриваются некоторые широко распространенные методы суммирования числовых рядов, изучаются условия тауберова типа (обозначаемые $T_{Q}(P)$-условиями) для этих методов.

Везде в дальнейшем $\left\{a_{n}\right\}_{n=0}^{+\infty}-$ последовательность действительных чисел, $\sum a_{n}-$ соответствующий ей ряд (когда пределы суммирования не указаны, мы считаем, что оно производится от 0 до $+\infty), k$ и $l$ - целые неотрицательные числа, $\alpha$ - действительное число.

Пусть $P$ и $Q$ - методы суммирования числовьх рядов. Условие $R$ на последовательность $\left\{a_{n}\right\}$ назьвается $T_{Q}(P)$-условием, если любой ряд $\sum a_{n}$, суммируемьй методом $P$ и такой, что $\left\{a_{n}\right\}$ удовлетворяет условию $R$, суммируем и методом $Q$. Метод $P$ при этом будем назьвать “верхним" методом, $Q$ - “нижним" методом.

Приведем в качестве примера лакунарные тауберовы условия для некоторых известных методов суммирования.

Пусть $\left\{n_{r}\right\}_{r=1}^{+\infty}-$ возрастающая последовательность натуральньх чисел, а последовательность $\left\{a_{n}\right\}$ такова, что

$$
a_{n}=0 \text { при } n \neq n_{1}, n_{2}, n_{3}, \ldots
$$

Тогда верны следующие утверждения.

ТЕорема А (определение метода Абеля $(A)$, см. [1, с. 20]). Eсли существует $q>1$ такое, что $n_{r+1} / n_{r} \geqslant q$ для всех $r$, то условие $(*)$ является $T_{(C, 0)}((A))$-условием $(c \mathcal{M} .[2])$.

Работа выполнена при финансовой поддержке Российского фонда фундаментальых исследований, грант № 97-01-00010, и программы “Ведущие научные школы”, грант № 96-15-96073. 
ТЕоремА Б (определение и основные свойства методов Чезаро $(C, \alpha)$ с $\alpha>-1$, см. $[1, \S 5.4, \S 5.5, \S 5.7])$. Если существует $q>1$ такое, что $n_{r+1} / n_{r} \geqslant q$ для всех $r$, то для любого $\alpha>0$ условие $(*)$ является $T_{(C, 0)}((C, \alpha))$-условием (следствие из теоремы A).

ТЕорема В (определение метода Бореля (B), см. [1, с. 107]). Если существует $d>0$ такое, что $\left(n_{r+1}-n_{r}\right) / \sqrt{n_{r}} \geqslant d$ для всех $r$, то условие $(*)$ является $T_{(C, 0)}((B))$-условием (см. $\left.[3]\right)$.

ТЕоремА Г (определение логарифмического метода $(L)$, см. $[1$, с. 108]). Ecли существует $\lambda>1$ такое, что $\ln n_{r+1} / \ln n_{r} \geqslant \lambda$ для всех $r$, то условие (*) является $T_{(C, 0)}((L))$-условием (см. [4]).

Тауберовы условия в этих результатах имеют один и тот же характер - между соседними ненулевыми элементами последовательности $\left\{a_{n}\right\}$ имеется “лакуна", величина которой зависит от "верхнего" метода.

В настоящей работе в роли "верхнего" и “нижнего" методов рассматриваются методы суммирования Чезаро различных порядков. Отметим, что когда "нижним" методом является $(C, 0)$, вопрос о лакунарном $T_{(C, 0)}((C, \alpha))$-условии решен окончательно, ибо, как вытекает из работы [5], требование на последовательность $\left\{n_{r}\right\}$, указанное в теореме Б, при любом $\alpha>0$ является не только достаточным, но и необходимым для того, чтобы условие $(*)$ было $T_{(C, 0)}((C, \alpha))$-условием. Поэтому нас будет интересовать случай, когда "нижний" метод есть метод $(C, k)$. Здесь будет установлена достаточность условия, в котором “лакуна" соответствующей величины разделяет не соседние ненулевые элементы, но ограниченные в совокупности множества из $k+1$ ненулевых элементов.

Переходя к точным формулировкам, дадим следующее определение.

Последовательность $\left\{a_{n}\right\}$ удовлетворяет условию $(G, k)$ (запись $\left\{a_{n}\right\} \in(G, k)$ ), если существуют натуральное число $C$, действительное число $q>1$ и последовательность натуральных чисел $\left\{n_{r}\right\}_{r=1}^{+\infty}$ такие, что

$$
\frac{n_{r+1}}{n_{r}} \geqslant q \text { для всех } r, \quad a_{n}=0, \quad \text { если } n \notin \bigcup_{r=1}^{+\infty}\left(n_{r}-C, n_{r}\right],
$$

и при каждом натуральном $r$ число отличных от 0 элементов $\left\{a_{n}\right\}$ с номерами $n \in$ $\left(n_{r}-C, n_{r}\right]$ не превьшает $k+1$.

Относительно введенных условий будет доказана теорема 1 , при $k=0$ совпадающая с теоремой Б.

Теорема 1. Пусть $k$ - фиксированное иелое число, $\alpha$ - фиксированное действительное число, причем $\alpha>k \geqslant 0$. Тогда требование $\left\{a_{n}\right\} \in(G, k)$ является $T_{(C, k)}((C, \alpha))$-условием.

В терминах условий $(G, k)$ приведенный результат является точным, т.е. справедливы следующие теоремы.

ТЕорема 2. Для любого иелого $k(k \geqslant 0)$ существует последовательность $\left\{a_{n}\right\}$ такая, что $\left\{a_{n}\right\} \in(G, k+1)$, ряд $\sum a_{n}$ суммируем методом $(C, \alpha)$ при любом $\alpha>k$, но ряд $\sum a_{n}$ не является суммируемым методом $(C, k)$. 
ТЕорема 3. Для любого иелого $k(k \geqslant 0)$ существует последовательность $\left\{a_{n}\right\}$ такая, что $\left\{a_{n}\right\} \in(G, k)$, ряд $\sum a_{n}$ суммируем методом $(C, k)$, но ни при каком $\omega$ $(0<\omega<k+1)$ ряд $\sum a_{n}$ не является суммируемым методом $(C, k-\omega)$.

В настоящей работе будет доказана теорема 1. Доказательства теорем 2 и 3 см. в [6, c. $90-100]$.

2. Установим некоторые вспомогательные утверждения, главным из которых будет лемма 5, имеющая, по мнению автора, самостоятельное значение.

Лемма 1. Пусть $k, i, l$ - фиксированные натуральные числа $(l<i), t$ - фиксированное целое число, $t \in[-k+1,1] ; R_{1}$ и $p$ - фиксированные действительные числа $\left(R_{1}>0\right)$. Тогда если

$$
\left|\sum_{\nu=0}^{l}(i-\nu+1)^{k+m} a_{\nu}\right|<R_{1}(i-l)^{m+p}
$$

для всех иелых $m \in[t, k+1]$, то

$$
\left|\sum_{\nu=0}^{l}(i-\nu+1)^{k+m}(l-\nu+1)^{m_{1}} a_{\nu}\right|<2^{m_{1}} R_{1}(i-l)^{m+m_{1}+p}
$$

для всех иелых $m$ из указанного выше промехутка и всех иельх $m_{1} \in[0, k+1-m]$.

ДокАЗАТЕЛЬСтво. Доказательство проведем индукцией по $m_{1}$. При $m_{1}=0$ требуемое неравенство вьполнено по условию.

Пусть утверждение леммы верно для $m_{1}=n_{1}$, где $n_{1}-$ целое, $n_{1} \in[0, k-t]$. Тогда неравенство

$$
\left|\sum_{\nu=0}^{l}(i-\nu+1)^{k+m}(l-\nu+1)^{n_{1}} a_{\nu}\right|<2^{n_{1}} R_{1}(i-l)^{m+n_{1}+p}
$$

справедливо для всех целых $m \in\left[t, k+1-n_{1}\right]$.

Отсюда для всех целых $m \in\left[t, k-n_{1}\right]$ вытекает

$$
\begin{aligned}
& \left|\sum_{\nu=0}^{l}(i-\nu+1)^{k+m}(l-\nu+1)^{n_{1}+1} a_{\nu}\right| \\
& \quad=\left|\sum_{\nu=0}^{l}(i-\nu+1)^{k+(m+1)}(l-\nu+1)^{n_{1}} a_{\nu}-(i-l) \sum_{\nu=0}^{l}(i-\nu+1)^{k+m}(l-\nu+1)^{n_{1}} a_{\nu}\right| \\
& \quad \leqslant\left|\sum_{\nu=0}^{l}(i-\nu+1)^{k+(m+1)}(l-\nu+1)^{n_{1}} a_{\nu}\right|+(i-l)\left|\sum_{\nu=0}^{l}(i-\nu+1)^{k+m}(l-\nu+1)^{n_{1}} a_{\nu}\right| \\
& \quad<2^{n_{1}} R_{1}(i-l)^{(m+1)+n_{1}+p}+(i-l) 2^{n_{1}} R_{1}(i-l)^{m+n_{1}+p}=2^{n_{1}+1} R_{1}(i-l)^{m+n_{1}+1+p} .
\end{aligned}
$$

Таким образом, из того, что утверждение леммы верно для $m_{1}=n_{1}$, где $n_{1}$ - целое, $n_{1} \in[0, k-t]$ (при этом $m$ - целое, $m \in\left[t, k+1-n_{1}\right]$ ), получаем его истинность для $m_{1}=n_{1}+1$ (при этом $m-$ целое, $m \in\left[t, k-n_{1}\right]$ ). Значит, утверждение леммы верно для любого целого $m_{1} \in[0, k-t+1]$ и любого целого $m \in\left[t, k-m_{1}+1\right]$ или, что то же самое, для любого целого $m \in[t, k+1]$ и любого целого $m_{1} \in[0, k+1-m]$. Лемма 1 доказана. 


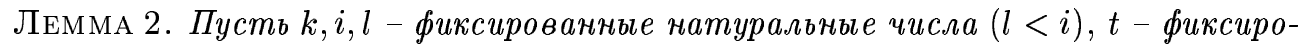
ванное челое число, $t \in[-k+1,1] ; R_{1}$ и $p$ - фиксированные действительные числа $\left(R_{1}>0\right)$. Тогда если

$$
\left|\sum_{\nu=0}^{l}(l-\nu+1)^{k+m} a_{\nu}\right|<R_{1}(i-l)^{m+p}
$$

для всех челых $m \in[t, k+1]$, то

$$
\left|\sum_{\nu=0}^{l}(l-\nu+1)^{k+m}(i-\nu+1)^{m_{1}} a_{\nu}\right|<2^{m_{1}} R_{1}(i-l)^{m+m_{1}+p}
$$

для всех челых $m$ из указанного выше промежутка и всех иельх $m_{1} \in[0, k+1-m]$.

Доказательство аналогично доказательству леммы 1.

ЛЕмма 3. Для любых натуральных чисел $k_{1}, k_{2}, i, l$, әде $i>l$, справедливо равенство

$$
\left(\sum_{\nu=0}^{l}(i-\nu+1)^{k_{1}} a_{\nu}\right)(i-l)^{k_{2}}=\sum_{j=0}^{k_{2}}(-1)^{j}\left(\begin{array}{c}
k_{2} \\
j
\end{array}\right) \sum_{\nu=0}^{l}(i-\nu+1)^{k_{1}+k_{2}-j}(l-\nu+1)^{j} a_{\nu} .
$$

ДокАЗАТЕЛЬСТво. Возьмем некоторое целое $\nu$ такое, что $0 \leqslant \nu \leqslant l$. Тогда

$$
\begin{aligned}
(i-\nu+1)^{k_{1}}(i-l)^{k_{2}} a_{\nu} & =a_{\nu}(i-\nu+1)^{k_{1}}((i-\nu+1)-(l-\nu+1))^{k_{2}} \\
& =a_{\nu} \sum_{j=0}^{k_{2}}(-1)^{j}\left(\begin{array}{c}
k_{2} \\
j
\end{array}\right)(i-\nu+1)^{k_{1}+k_{2}-j}(l-\nu+1)^{j} .
\end{aligned}
$$

Просуммировав полученное равенство по $\nu$ от 0 до $l$ и изменив затем порядок суммирования, установим требуемое. Лемма 3 доказана.

ЛЕмма 4. Для любых натуральных чисел $k_{1}, k_{2}, i, l$, әде $i>l$, справедливо равенство

$$
\left(\sum_{\nu=0}^{l}(l-\nu+1)^{k_{1}} a_{\nu}\right)(i-l)^{k_{2}}=\sum_{j=0}^{k_{2}}(-1)^{k_{2}-j}\left(\begin{array}{c}
k_{2} \\
j
\end{array}\right) \sum_{\nu=0}^{l}(l-\nu+1)^{k_{1}+k_{2}-j}(i-\nu+1)^{j} a_{\nu} .
$$

Доказательство аналогично доказательству леммы 3.

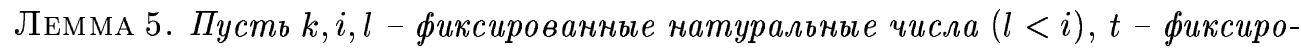
ванное челое число, $t \in[-k+1,1] ; R_{2}$ и $p$ - фиксированные действительные числа $\left(R_{2}>0\right)$. Тогда если

$$
\left|\sum_{\nu=0}^{l}(i-\nu+1)^{k+m} a_{\nu}\right|<R_{2}(i-l)^{m+p}, \quad\left|\sum_{\nu=0}^{l}(l-\nu+1)^{k+m} a_{\nu}\right|<R_{2}(i-l)^{m+p}
$$

для всех иельх $m \in[t, k+1]$, mо

$$
\begin{aligned}
& \left|\sum_{\nu=0}^{l}(i-\nu+1)^{k+t-1} a_{\nu}\right|<2^{3 k+1} R_{2}(i-l)^{t+p-1} \\
& \left|\sum_{\nu=0}^{l}(l-\nu+1)^{k+t-1} a_{\nu}\right|<2^{3 k+1} R_{2}(i-l)^{t+p-1} .
\end{aligned}
$$


ДокАЗАТЕЛЬСТво. Согласно леммам 1 и 2 справедливы оценки

$$
\begin{aligned}
& \left|\sum_{\nu=0}^{l}(i-\nu+1)^{k+m}(l-\nu+1)^{m_{1}} a_{\nu}\right|<2^{2 k} R_{2}(i-l)^{m+m_{1}+p}, \\
& \left|\sum_{\nu=0}^{l}(l-\nu+1)^{k+m}(i-\nu+1)^{m_{1}} a_{\nu}\right|<2^{2 k} R_{2}(i-l)^{m+m_{1}+p} .
\end{aligned}
$$

Неравенства (1) верны для всех целых $m \in[t, k+1]$ и всех целых $m_{1} \in[0, k+1-m]$. В частности, при $m=t$ и $m_{1}=k+t-1$ имеем

$$
\begin{aligned}
& \left|\sum_{\nu=0}^{l}(i-\nu+1)^{k+t}(l-\nu+1)^{k+t-1} a_{\nu}\right|<2^{2 k} R_{2}(i-l)^{k+2 t+p-1}, \\
& \left|\sum_{\nu=0}^{l}(l-\nu+1)^{k+t}(i-\nu+1)^{k+t-1} a_{\nu}\right|<2^{2 k} R_{2}(i-l)^{k+2 t+p-1} .
\end{aligned}
$$

Далее, с помощью соотношений (2) получаем

$$
\begin{aligned}
& \left|\sum_{\nu=0}^{l}(i-\nu+1)^{k+t-1}(l-\nu+1)^{k+t-1} a_{\nu}\right| \\
& \quad=\frac{1}{i-l}\left|\sum_{\nu=0}^{l}(i-\nu+1)^{k+t}(l-\nu+1)^{k+t-1} a_{\nu}-\sum_{\nu=0}^{l}(l-\nu+1)^{k+t}(i-\nu+1)^{k+t-1} a_{\nu}\right| \\
& \quad<2^{2 k+1} R_{2}(i-l)^{k+2 t+p-2} .
\end{aligned}
$$

Если в условии леммы $t=-k+1$, то из неравенства (3) сразу вытекает требуемое утверждение. Продолжим доказательство в случае, когда $t$ принимает фиксированное целое значение в промежутке $[-k+2,1]$.

Положим $m_{1}=n_{1}, m=k+2 t-2-n_{1}$, где $n_{1}$ - целое число, $n_{1} \in[0, k+t-2]$. Легко заметить, что $m \in[t, k+1], m_{1} \in[0, k+1-m]$. Следовательно, для указанных $m$ и $m_{1}$ оценки (1) вьполняются и имеют вид

$$
\begin{aligned}
& \left|\sum_{\nu=0}^{l}(i-\nu+1)^{2 k+2 t-2-n_{1}}(l-\nu+1)^{n_{1}} a_{\nu}\right|<2^{2 k} R_{2}(i-l)^{k+2 t-2+p}, \\
& \left|\sum_{\nu=0}^{l}(l-\nu+1)^{2 k+2 t-2-n_{1}}(i-\nu+1)^{n_{1}} a_{\nu}\right|<2^{2 k} R_{2}(i-l)^{k+2 t-2+p} .
\end{aligned}
$$

Неравенства (4) и (5) верны для любого целого $n_{1} \in[0, k+t-2]$. Применим лемму $3 \mathrm{c}$ $k_{1}=k+t-1$ и $k_{2}=k+t-1$. Согласно этой лемме имеем

$$
\begin{aligned}
& \left(\sum_{\nu=0}^{l}(i-\nu+1)^{k+t-1} a_{\nu}\right)(i-l)^{k+t-1} \\
& \quad=\sum_{j=0}^{k+t-1}(-1)^{j}\left(\begin{array}{c}
k+t-1 \\
j
\end{array}\right) \sum_{\nu=0}^{l}(i-\nu+1)^{2 k+2 t-2-j}(l-\nu+1)^{j} a_{\nu} .
\end{aligned}
$$


Оценив теперь модуль суммы суммой модулей и воспользовавшись неравенствами (3) и (4), получим

$$
\begin{aligned}
& \left|\sum_{\nu=0}^{l}(i-\nu+1)^{k+t-1} a_{\nu}\right| \\
& \quad \leqslant \frac{1}{(i-l)^{k+t-1}}\left(\sum_{n_{1}=0}^{k+t-2}\left(\begin{array}{c}
k+t-1 \\
n_{1}
\end{array}\right)\left|\sum_{\nu=0}^{l}(i-\nu+1)^{2 k+2 t-2-n_{1}}(l-\nu+1)^{n_{1}} a_{\nu}\right|\right. \\
& \left.\quad+\left(\begin{array}{c}
k+t-1 \\
k+t-1
\end{array}\right)\left|\sum_{\nu=0}^{l}(i-\nu+1)^{k+t-1}(l-\nu+1)^{k+t-1} a_{\nu}\right|\right) \\
& \left.\quad<\left(\begin{array}{c}
k+t-2 \\
\sum_{n_{1}=0}^{k+t-1}
\end{array}\right)\right) 2^{2 k} R_{2}(i-l)^{t+p-1}+2^{2 k+1} R_{2}(i-l)^{t+p-1} \\
& \quad<2^{3 k+1} R_{2}(i-l)^{t+p-1} .
\end{aligned}
$$

Аналогично, используя лемму 4 с $k_{1}=k+t-1, k_{2}=k+t-1$ и соотношения (3) и (5), вьводим неравенство

$$
\left|\sum_{\nu=0}^{l}(l-\nu+1)^{k+t-1} a_{\nu}\right|<2^{3 k+1} R_{2}(i-l)^{t+p-1} .
$$

Неравенства (6) и (7) составляют заключение леммы. Лемма 5 доказана.

3. ДоКАЗАТЕЛЬСТво ТЕОРЕмы 1 . Как было отмечено вьше, при $k=0$ утверждение теоремы известно. Проведем доказательство для натурального $k$.

Согласно определению условия $(G, k)$ обладают следующим свойством: если $\left\{a_{n}\right\} \in$ $(G, k)$, то $\left\{a_{n}\right\} \in(G, l)$, где $l$ - любое целое число большее $k$. Отсюда и из того, что ряд $\sum a_{n}$, суммируемый методом $(C, \gamma)$, суммируем любым методом $(C, \beta)$ с $\beta>\gamma>-1$, вытекает, что для доказательства теоремы нам достаточно установить ее в случае $\alpha=k+1$.

Итак, пусть $k$ - фиксированное натуральное число, $\left\{a_{n}\right\}$ - произвольная последовательность такая, что $\left\{a_{n}\right\} \in(G, k)$ и $\sum a_{n}=A((C, k+1))$ (запись $\sum a_{n}=A(P)$ обозначает, что ряд $\sum a_{n}$ суммируем к числу $A$ методом $\left.P\right)$.

Докажем, что $\sum a_{n}=A((C, k))$.

Так как $\left\{a_{n}\right\} \in(G, k)$, существуют натуральное число $C$, действительное число $q>1$ и последовательность натуральных чисел $\left\{n_{r}\right\}_{r=1}^{+\infty}$ такие, что

$$
\frac{n_{r+1}}{n_{r}} \geqslant q \text { для всех } r, \quad a_{n}=0, \text { если } n \notin \bigcup_{r=1}^{+\infty}\left(n_{r}-C, n_{r}\right],
$$

и при каждом натуральном $r$ число отличных от 0 элементов $\left\{a_{n}\right\}$ с номерами $n \in$ $\left(n_{r}-C, n_{r}\right]$ не превьшает $k+1$.

Изменяя, если это необходимо, конечное число членов последовательности $\left\{a_{n}\right\}$, мы можем без ограничения общности считать, что $A=0$, а числа $C, q$ и последовательность $\left\{n_{r}\right\}$ выбраны таким образом, что $C>k+1, q \leqslant n_{r+1} / n_{r} \leqslant q^{3}<2$ для всех $r$. 
Приведем известный результат для методов суммирования Чезаро (см., например, $\left[1\right.$, c. 146-147]). Если $\sum a_{n}=0((C, s))$, где $s$ - некоторое целое неотрицательное число, то

$$
\left|\sum_{\nu=0}^{n}(n-\nu+1)^{s} a_{\nu}\right|=o\left(n^{s}\right) .
$$

Тогда, поскольку из суммируемости ряда $\sum a_{n}$ к 0 методом $(C, k+1)$ следует его суммируемость к 0 методами $(C, k+2), \ldots,(C, 2 k+1)$, то для любого целого $m \in[1, k+1]$ справедливо соотношение

$$
\left|\sum_{\nu=0}^{n}(n-\nu+1)^{k+m} a_{\nu}\right|=o\left(n^{k+m}\right) .
$$

Возьмем любое фиксированное $\varepsilon>0$. Для этого $\varepsilon$ согласно (8) существует $N$ такое, что для любого $n \geqslant N$ и для любого целого $m \in[1, k+1]$ выполнено неравенство

$$
\left|\sum_{\nu=0}^{n}(n-\nu+1)^{k+m} a_{\nu}\right|<\varepsilon(\sqrt[8]{q}-1)^{2 k+1} n^{k+m} .
$$

Выберем в качестве $r_{0}$ наименьший из номеров $r$ последовательности $\left\{n_{r}\right\}$ такой, что $n_{r_{0}}>N$ и

$$
n_{r_{0}}>\frac{C}{\sqrt[8]{q}-1} .
$$

Пусть $n_{r}-$ любой фиксированньй член последовательности $\left\{n_{r}\right\}$ такой, что $n_{r}>n_{r_{0}}$, a $l$ и $i$ - любые фиксированные натуральные числа такие, что

$$
n_{r} \leqslant l<\sqrt[8]{q} \cdot l \leqslant i \leqslant n_{r+1}-C<2 n_{r} .
$$

Пользуясь формулами (9) и (11), а также тем, что $a_{n}=0$ при $n \in\left(n_{r}, n_{r+1}-C\right]$, получим оценку

$$
\begin{aligned}
\left|\sum_{\nu=0}^{l}(i-\nu+1)^{k+m} a_{\nu}\right| & =\left|\sum_{\nu=0}^{i}(i-\nu+1)^{k+m} a_{\nu}\right|<\varepsilon(\sqrt[8]{q}-1)^{2 k+1} i^{k+m} \\
& <2^{k+m} \varepsilon(\sqrt[8]{q}-1)^{k+1-m}(\sqrt[8]{q}-1)^{k+m} l^{k+m} \leqslant 2^{k+m} \varepsilon(i-l)^{k+m} .
\end{aligned}
$$

Положим $M_{1}=2^{2 k+1}$ ( $M_{1}$ - константа, так как $k$ - фиксированная постоянная). Тогда для всех целых $m \in[1, k+1]$ справедливо неравенство

$$
\left|\sum_{\nu=0}^{l}(i-\nu+1)^{k+m} a_{\nu}\right|<M_{1} \varepsilon(i-l)^{k+m} .
$$

Аналогично, для тех же $m$ вьполнено

$$
\left|\sum_{\nu=0}^{l}(l-\nu+1)^{k+m} a_{\nu}\right|<M_{1} \varepsilon(i-l)^{k+m} .
$$


Заметим, что поскольку верны оценки (12) и (13), то вьполнены условия леммы 5 , где величины $i, l, k$ такие же, как в нашей теореме, $p=k, t=1, R_{2}=M_{1} \varepsilon$.

Тогда можно сделать вьвод, что

$$
\left|\sum_{\nu=0}^{l}(i-\nu+1)^{k} a_{\nu}\right|<2^{3 k+1} M_{1} \varepsilon(i-l)^{k}, \quad\left|\sum_{\nu=0}^{l}(l-\nu+1)^{k} a_{\nu}\right|<2^{3 k+1} M_{1} \varepsilon(i-l)^{k} .
$$

Заметим, что поскольку верны оценки (12)-(14), то выполнены условия леммы 5 , где величины $i, l, k$ такие же, как в нашей теореме, $p=k, t=0, R_{2}=2^{3 k+1} M_{1} \varepsilon$.

Тогда можно сделать вьвод, что

$$
\begin{aligned}
& \left|\sum_{\nu=0}^{l}(i-\nu+1)^{k-1} a_{\nu}\right|<\left(2^{3 k+1}\right)^{2} M_{1} \varepsilon(i-l)^{k-1}, \\
& \left|\sum_{\nu=0}^{l}(l-\nu+1)^{k-1} a_{\nu}\right|<\left(2^{3 k+1}\right)^{2} M_{1} \varepsilon(i-l)^{k-1} .
\end{aligned}
$$

И так далее, применяя поочередно лемму 5 (при очередном использовании леммы 5 все фигурирующие в условии величины, кроме $t$ и $R_{2}$, остаются прежними; $t$ уменьшается на 1 , а $R_{2}$ увеличивается в $2^{3 k+1}$ раз) до тех пор, пока $t$ не станет равным $-k+1$ (при $t=-k+1$ лемма 5 применяется в последний раз), получим следующие неравенства, верные для всех целых $s \in[0, k]$ :

$$
\begin{aligned}
& \left|\sum_{\nu=0}^{l}(i-\nu+1)^{k-s} a_{\nu}\right|<\left(2^{3 k+1}\right)^{s+1} M_{1} \varepsilon(i-l)^{k-s}, \\
& \left|\sum_{\nu=0}^{l}(l-\nu+1)^{k-s} a_{\nu}\right|<\left(2^{3 k+1}\right)^{s+1} M_{1} \varepsilon(i-l)^{k-s} .
\end{aligned}
$$

Используя то, что $a_{n}=0$ при $n \in(l, i]$, перепишем $(15)$ в виде

$$
\left|\sum_{\nu=0}^{i}(i-\nu+1)^{k-s} a_{\nu}\right|<\left(2^{3 k+1}\right)^{s+1} M_{1} \varepsilon(i-l)^{k-s}
$$

Пусть теперь $n$ - любое целое число из отрезка $\left[n_{r}, n_{r+1}-C\right]$. Тогда выполнено хотя бы одно из двух неравенств:

i) $\sqrt[8]{q} \cdot n_{r} \leqslant n$, или

ii) $\sqrt[8]{q} \cdot n \leqslant n_{r+1}-C$.

Действительно, если бы оба эти неравенства были бы неверны, то имели бы $\sqrt[4]{q} \cdot n_{r}>$ $\sqrt[8]{q} \cdot n>n_{r+1}-C \geqslant q \cdot n_{r}-C$. И тогда $n_{r_{0}}<n_{r}<C /(q-\sqrt[4]{q})=C /\left(\sqrt[4]{q}\left(q^{3 / 4}-1\right)\right)<$ $C /(\sqrt[8]{q}-1)$, что противоречит неравенству $(10)$.

Пусть выполнено і). В этом случае мы можем рассмотреть в качестве $l$ число $n_{r}$, a в качестве $i$ - число $n$ (так как для таких $l$ и $i$ вьполнены соотношения (11)). Тогда из неравенства (17) получаем

$$
\left|\sum_{\nu=0}^{n}(n-\nu+1)^{k-s} a_{\nu}\right|<\left(2^{3 k+1}\right)^{s+1} M_{1} \varepsilon n^{k-s} .
$$


Если же выполнено іi), то мы можем рассмотреть в качестве $l$ число $n$, а в качестве $i$ - число $n_{r+1}-C$ (так как для таких $l$ и $i$ выполнены соотношения (11)). Тогда из неравенств (11) и (16) получаем

$$
\left|\sum_{\nu=0}^{n}(n-\nu+1)^{k-s} a_{\nu}\right|<\left(2^{3 k+1}\right)^{s+1} M_{1} \varepsilon n^{k-s} .
$$

Тем самым, мы показали, что для любого целого $r, r>r_{0}$, для любого целого $n$, $n \in\left[n_{r}, n_{r+1}-C\right]$, и для всех цельх $s, s \in[0, k]$, справедливо неравенство

$$
\left|\sum_{\nu=0}^{n}(n-\nu+1)^{k-s} a_{\nu}\right|<\left(2^{3 k+1}\right)^{s+1} M_{1} \varepsilon n^{k-s} .
$$

Обозначим $M_{2}=\left(2^{3 k+1}\right)^{k+2} \cdot M_{1}, p=k-s$. Положив в $(18) n=n_{r+1}$ и $n=n_{r+1}-C$, получим следующие оценки, справедливые при всех целых $r, r>r_{0}$, и всех целых $p$, $p \in[0, k]:$

$$
\begin{array}{r}
\left|\sum_{\nu=0}^{n_{r+1}}\left(n_{r+1}-\nu+1\right)^{p} a_{\nu}\right|<M_{2} \varepsilon n_{r}^{p}, \\
\left|\sum_{\nu=0}^{n_{r+1}-C}\left(\left(n_{r+1}-C\right)-\nu+1\right)^{p} a_{\nu}\right|<M_{2} \varepsilon n_{r}^{p} .
\end{array}
$$

Обозначим через $\gamma_{r j}$ (где $r$ - целое, $r>r_{0}, j$ - целое, $0 \leqslant j \leqslant k$ ) некоторые различные числа, лежащие в промежутке $\left(n_{r+1}-C, n_{r+1}\right]$, таким образом, чтобы в число $\gamma_{r j}$ попали все номера $n$ такие, что $a_{n} \neq 0$ и $n \in\left(n_{r+1}-C, n_{r+1}\right]$. Таких номеров $n$ в каждом из промежутков не более чем $k+1$. Если количество таких номеров в каком-либопромежутке $\left(n_{r+1}-C, n_{r+1}\right]$ строго меньше чем $k+1$, то в качестве оставшихся элементов $\gamma_{r j}$ (при фиксированном $r$ ) мы возьмем необходимое количество чисел из $\left(n_{r+1}-C, n_{r+1}\right]$ произвольным образом (сделать это возможно, так как $C>k+1)$. Для определенности будем считать, что $\gamma_{r 0}<\gamma_{r 1}<\cdots<\gamma_{r k}$.

Обозначим также через $\beta_{r j}$ разность между $n_{r+1}+1$ и $\gamma_{r j}$, т.е. $\beta_{r j}=n_{r+1}+1-\gamma_{r j}$ при всех целых $r, r>r_{0}$, и всех целых $j, 0 \leqslant j \leqslant k$.

Поскольку $\gamma_{r j}$ - различные и целые, $\gamma_{r j} \in\left(n_{r+1}-C, n_{r+1}\right]$, то $\beta_{r j}$-целые, $\beta_{r j} \in[1, C]$, $\beta_{\text {ri }} \neq \beta_{\text {rj }}$ при $i \neq j$.

$\mathrm{C}$ использованием введенных обозначений можно записать

$$
\begin{aligned}
& \sum_{\nu=0}^{n_{r+1}}\left(n_{r+1}-\nu+1\right)^{p} a_{\nu} \\
& \quad=\sum_{\nu=0}^{n_{r+1}-C}\left(\left(n_{r+1}-C\right)-\nu+1+C\right)^{p} a_{\nu}+\sum_{\nu=n_{r+1}-C+1}^{n_{r+1}}\left(n_{r+1}-\nu+1\right)^{p} a_{\nu} \\
& \quad=\sum_{\nu=0}^{n_{r+1}-C} \sum_{i=0}^{p}\left(\begin{array}{c}
p \\
i
\end{array}\right) C^{i}\left(\left(n_{r+1}-C\right)-\nu+1\right)^{p-i} a_{\nu}+\sum_{j=0}^{k}\left(n_{r+1}-\gamma_{r j}+1\right)^{p} a_{\gamma_{r j}} \\
& \quad=\sum_{i=0}^{p}\left(\begin{array}{c}
p \\
i
\end{array}\right) C^{i}\left(\begin{array}{c}
n_{r+1}-C \\
\sum_{\nu=0}^{k} \beta_{r j}^{p} a_{\gamma_{r j} .} .
\end{array}\right.
\end{aligned}
$$


Положим

$$
b_{r p}=\sum_{\nu=0}^{n_{r+1}}\left(n_{r+1}-\nu+1\right)^{p} a_{\nu}-\sum_{i=0}^{p}\left(\begin{array}{c}
p \\
i
\end{array}\right) C^{i}\left(\sum_{\nu=0}^{n_{r+1}-C}\left(\left(n_{r+1}-C\right)-\nu+1\right)^{p-i} a_{\nu}\right)
$$

Тогда равенство (21), справедливое для всех целых $r, r>r_{0}$, и всех целых $p, p \in[0, k]$, примет вид

$$
\sum_{j=0}^{k} \beta_{r j}^{p} a_{\gamma_{r j}}=b_{r p}
$$

Оценим теперь величину $\left|b_{r p}\right|$ с помощью (19), (20), (22):

$$
\begin{aligned}
\left|b_{r p}\right| & \leqslant\left|\sum_{\nu=0}^{n_{r+1}}\left(n_{r+1}-\nu+1\right)^{p} a_{\nu}\right|+\sum_{i=0}^{p}\left(\begin{array}{c}
p \\
i
\end{array}\right) C^{i}\left|\sum_{\nu=0}^{n_{r+1}-C}\left(\left(n_{r+1}-C\right)-\nu+1\right)^{p-i} a_{\nu}\right| \\
& <M_{2} \varepsilon n_{r}^{p}+\sum_{i=0}^{p}\left(\begin{array}{c}
p \\
i
\end{array}\right) C^{i} M_{2} \varepsilon n_{r}^{p} \leqslant\left(2^{k} C^{k}+1\right) M_{2} \varepsilon n_{r}^{p} .
\end{aligned}
$$

Полагая $M_{3}=\left(2^{k} C^{k}+1\right) M_{2}$, получаем, что для всех целых $r, r>r_{0}$, и всех целых $p$, $p \in[0, k]$, верна оценка

$$
\left|b_{r p}\right|<M_{3} \varepsilon n_{r}^{p}
$$

Зафиксируем теперь произвольное целое $r, r>r_{0}$, и рассмотрим систему из $k+1$ линейных уравнений с неизвестными $f_{0}, f_{1}, \ldots, f_{k}$ :

$$
\begin{array}{r}
f_{0}+f_{1}+\cdots+f_{k}=b_{r 0}, \\
\beta_{r 0} f_{0}+\beta_{r 1} f_{1}+\cdots+\beta_{r k} f_{k}=b_{r 1}, \\
\beta_{r 0}^{2} f_{0}+\beta_{r 1}^{2} f_{1}+\cdots+\beta_{r k}^{2} f_{k}=b_{r 2}, \\
\cdots \cdots \cdots+\cdots \cdots+\cdots \cdots+\cdots \cdots+\beta_{r k}^{k} f_{k}=b_{r k} . \\
\beta_{r 0}^{k} f_{0}+\beta_{r 1}^{k} f_{1}+\cdots+
\end{array}
$$

Одно решение этой системы мы знаем, а именно, согласно $(23)$ набор $\left(f_{0}, f_{1}, \ldots, f_{k}\right)=$ $\left(a_{\gamma_{r 0}}, a_{\gamma_{r 1}}, \ldots, a_{\gamma_{r k}}\right)$ является решением.

Можно, однако, подойти к вопросу решения системы (25) с другой стороны. Определитель матрицы системы (25)

$$
d=\left|\begin{array}{cccc}
1 & 1 & \ldots & 1 \\
\beta_{r 0} & \beta_{r 1} & \ldots & \beta_{r k} \\
\beta_{r 0}^{2} & \beta_{r 1}^{2} & \ldots & \beta_{r k}^{2} \\
\vdots & \vdots & \ddots & \vdots \\
\beta_{r 0}^{k} & \beta_{r 1}^{k} & \ldots & \beta_{r k}^{k}
\end{array}\right|
$$

является определителем Вандермонда и равен произведению всевозможных разностей $\beta_{r j}-\beta_{r i}$, где $0 \leqslant i<j \leqslant k$.

Поскольку все $\beta_{r j}$ - целые и $\beta_{r i} \neq \beta_{r j}$ при $i \neq j$, то $\left|\beta_{r j}-\beta_{r i}\right| \geqslant 1$ для $0 \leqslant i<j \leqslant k$. 
Отсюда получаем

$$
|d|=\prod_{0 \leqslant i<j \leqslant k}\left|\beta_{r j}-\beta_{r i}\right| \geqslant 1 .
$$

Таким образом, определитель системы (25) отличен от 0 и, следовательно, согласно общей теории систем линейных уравнений система (25) обладает единственным решением, которое находится по формулам $f_{j}=d_{j} / d(j=0,1, \ldots, k)$, где $d_{j}$ - определитель, получающийся из определителя $d$ заменой $(j+1)$-го столбца в $d$ на столбец свободных членов

$$
\left(\begin{array}{c}
b_{r 0} \\
\vdots \\
b_{r k}
\end{array}\right) \text {. }
$$

Принимая во внимание оценку (24) и неравенства $\left|\beta_{r j}\right| \leqslant C(j=0,1, \ldots, k)$, легко заметить, что

$$
\left|d_{j}\right|<(k+1) ! \cdot\left(C^{k}\right)^{k} \cdot M_{3} \varepsilon n_{r}^{k}, \quad j=0,1, \ldots, k .
$$

Положим $M_{4}=(k+1) ! \cdot\left(C^{k}\right)^{k} \cdot M_{3}$. Тогда, пользуясь неравенствами $(26)$ и $(27)$, получаем

$$
\left|f_{j}\right|=\frac{\left|d_{j}\right|}{|d|}<M_{4} \varepsilon n_{r}^{k}
$$

Но, как было отмечено ранее, единственное решение системы (25) реализуется на наборе $\left(a_{\gamma_{r 0}}, a_{\gamma_{r 1}}, \ldots, a_{\gamma_{r k}}\right)$, т.е. $f_{j}=a_{\gamma_{r j}}$. Следовательно, для любого целого $r, r>r_{0}$, и любого целого $j, j \in[0, k]$, справедлива оценка

$$
\left|a_{\gamma_{r j}}\right|<M_{4} \varepsilon n_{r}^{k}
$$

Пусть теперь $n$ - любое целое, $n \in\left(n_{r+1}-C, n_{r+1}\right)$, где $r$ - некоторое целое, $r>r_{0}$. Тогда, как следует из формул $(20)$ и $(28)$, для любого целого $p, p \in[0, k]$, выполнены соотношения

$$
\begin{aligned}
\left|\sum_{\nu=0}^{n}(n-\nu+1)^{p} a_{\nu}\right| \leqslant & \left|\sum_{\nu=0}^{n_{r+1}-C}\left(\left(n-n_{r+1}+C\right)+\left(\left(n_{r+1}-C\right)-\nu+1\right)\right)^{p} a_{\nu}\right| \\
& +\left|\sum_{\nu=n_{r+1}-C+1}^{n}(n-\nu+1)^{p} a_{\nu}\right| \\
\leqslant & \left|\sum_{\nu=0}^{n_{r+1}-C} \sum_{i=0}^{p}\left(\begin{array}{c}
p \\
i
\end{array}\right)\left(n-n_{r+1}+C\right)^{i}\left(\left(n_{r+1}-C\right)-\nu+1\right)^{p-i} a_{\nu}\right| \\
& +\sum_{\nu=n_{r+1}-C+1}^{n}(n-\nu+1)^{p}\left|a_{\nu}\right| \\
\leqslant & \sum_{i=0}^{p}\left(\begin{array}{c}
p \\
i
\end{array}\right) C^{i} \sum_{\nu=0}^{n_{r+1}-C}\left(\left(n_{r+1}-C\right)-\nu+1\right)^{p-i} a_{\nu}\left|+C^{p} \sum_{j=0}^{k}\right| a_{\gamma_{r j}} \mid \\
< & C^{p} 2^{p} M_{2} \varepsilon n_{r}^{p}+C^{p}(k+1) M_{4} \varepsilon n_{r}^{k} \leqslant C^{k}\left(2^{k} M_{2}+(k+1) M_{4}\right) \varepsilon n_{r}^{k} .
\end{aligned}
$$


Положим $M_{5}=C^{k}\left(2^{k} M_{2}+(k+1) M_{4}\right)$.

Объединяя оценки (18) и (29), заключаем, что для любого целого $n, n \geqslant n_{r_{0}+1}$, и для любого целого $p, p \in[0, k]$, справедливо неравенство

$$
\left|\sum_{\nu=0}^{n}(n-\nu+1)^{p} a_{\nu}\right|<M_{5} \varepsilon n^{k} .
$$

Рассмотрим чезаровские средние для ряда $\sum a_{n}[1$, с. $125-126]$ :

$$
C_{n}^{k}=\frac{1}{(n+1) \cdots(n+k)} \sum_{\nu=0}^{n}(n-\nu+1)(n-\nu+2) \cdots(n-\nu+k) \cdot a_{\nu}
$$

Полагая $M=2^{k-1}(k-1) ! M_{5}$ и используя оценку $(30)$, для любого целого $n \geqslant n_{r_{0}+1}$ получим

$$
\begin{aligned}
\left|C_{n}^{k}\right| & =\frac{1}{(n+1) \cdots(n+k)}\left|\sum_{\nu=0}^{n}(n-\nu+1)(n-\nu+1+1) \cdots(n-\nu+1+k-1) \cdot a_{\nu}\right| \\
& \leqslant \frac{(k-1) !}{(n+1) \cdots(n+k)} \sum_{i=1}^{k}\left(\begin{array}{c}
k-1 \\
i-1
\end{array}\right) \cdot\left|\sum_{\nu=0}^{n}(n-\nu+1)^{i} a_{\nu}\right| \\
& \leqslant \frac{2^{k-1}(k-1) !}{n^{k}} M_{5} \varepsilon n^{k}=M \varepsilon .
\end{aligned}
$$

Тем самьм, мы показали, что

$$
\lim _{n \rightarrow+\infty} C_{n}^{k}=0
$$

т.е. $\sum a_{n}=0((C, k))$. Теорема 1 доказана.

Автор глубоко признателен научному руководителю член-корреспонденту РАН П. Л. Ульянову за внимание к работе и ряд полезных замечаний.

\section{СПИСОК ЦИТИРОВАННОЙ ЛИТЕРАТУРЫ}

[1] Харди Г. Расходящиеся ряды. М.: ИЛ, 1951.

[2] Hardy G. H., Littlewood J. E. A further note on the converse of Abel's theorem // Proc. London Math. Soc. 1926. V. 25. № 2. P. 219-236.

[3] Мельник В.И. Тауберова теорема о "больших показателях" для метода суммирования Бореля // Матем. сб. 1965. Т. 68. № 1. С. 17-25.

[4] Krishnan V.K. Gap Tauberian theorem for logarithmic summability $(L) / /$ Quart J. Math. Oxford. Ser. (2). 1979. V. 30. № 2. P. 77-87.

[5] Rajagopal C. T. On the relation of limitation theorems to high-indices theorems // J. London Math. Soc. 1953. V. 28. P. 322-329.

[6] Степанянц С. А. Условия тауберова типа, связывающие различные методы суммирования. Дисс. ... к. ф.-м.н. М.: МГУ, 1996. 